\title{
Differences in muscle coactivation during postural control between healthy older and young adults.
}

AUTHOR(S):

Nagai, Koutatsu; Yamada, Minoru; Uemura, Kazuki; Yamada, Yosuke; Ichihashi, Noriaki; Tsuboyama, Tadao

\section{CITATION:}

Nagai, Koutatsu ... [et al]. Differences in muscle coactivation during postural control between healthy older and young adults.. Archives of gerontology and geriatrics 2011 , 53(3): 338-343

ISSUE DATE:

2011-11

URL:

http://hdl.handle.net/2433/147242

\section{RIGHT:}

(c) 2011 Elsevier Ireland Ltd.; この論文は出版社版でありません。引用の 際には出版社版をご確認ご利用ください。; This is not the published version. Please cite only the published version. 
Differences in muscle coactivation during postural control between healthy older and young adults

\section{Koutatsu Nagai ${ }^{a, *}$, Minoru Yamada $^{a}$, Kazuki Uemura $^{a}$, Yosuke Yamada ${ }^{b}$, Noriaki Ichihashi ${ }^{a}$, Tadao Tsuboyama ${ }^{a}$}

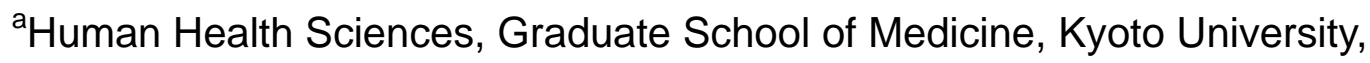
53, Kawahara-cho, Shogoin, Sakyo-ku, Kyoto, 606-8507, Japan

\footnotetext{
${ }^{\mathrm{b}}$ The Fukuoka University Institute for Physical Activity, 1-19-8, Nanakuma, Jyonanku, Fukuoka, 814-0180, Japan
}

*Corresponding author:

Phone: +(81-75)-751-3935

Fax: $+(81-75)-751-3948$

E-mail:k.nagai@ky2.ecs.kyoto-u.ac.jp 


\section{Abstract}

The purpose of this study was to clarify the difference in muscle coactivation during postural control between older and young adults and to identify the characteristics of postural control strategies in older adults by investigating the relationship between muscle coactivation and postural control ability. Forty-six healthy older adults ( $82.0 \pm 7.5$ years) and 34 healthy young adults $(22.1 \pm 2.3$ years) participated. The postural tasks selected consisted of static standing, functional reach, functional stability boundary and gait. Coactivation of the ankle joint was recorded during each task via electromyography (EMG). The older adults showed significantly higher coactivation than the young adults during the tasks of standing, functional reach, functional stability boundary (forward), and gait $(p<0.01)$. Postural sway area $(\rho=0.42, p<0.05)$ and functional reach distance $(\rho=-0.52, p<0.05)$ significantly correlated with coactivation during the corresponding task in older adults, i.e., muscle coactivation was significantly higher in the elderly with low postural control ability than in the elderly with high balance ability. Increased muscle coactivation could be a necessary change to compensate for a deterioration in postural control accompanying healthy aging. Further research is needed to clarify in greater detail positive and negative effects of muscle coactivation on postural control.

Keywords: Coactivation, electromyography, older adults, postural control

\section{Introduction}

Appropriate temporal separation between agonist and antagonist activation of muscles has been observed for well-controlled voluntary movements (Fujii et al., 2009). In clinical situations, however, this separation is attenuated, muscle coactivation is increased, and motor control becomes poor (Dierick et al., 2002). Even with normal aging, greater coactivation is induced during single-joint movements (Klein et al., 2001; Macaluso et al., 2002) and gait (Mian et al., 2006; Hortobagyi et al., 2009) in the elderly. Greater muscle coactivation, in turn, increases the metabolic cost of gait (Mian et al., 2006), which can cause fatigue and shorten activity duration.

Although several studies have reported age-associated increases in muscle coactivation during dynamic movements (Schmitz et al., 2008; Hortobagyi et al., 2009), limited information is available regarding muscle coactivation under static 
postural control (Melzer et al., 2001). Increased muscle coactivation in older subjects is most commonly described as a compensatory mechanism to increase joint stiffness that thereby enhances stability (Baratta et al., 1988; Solomonow et al., 1988; Hortobagyi and DeVita, 2000). Older adults may walk with high muscle coactivation as a balance-maintaining strategy in response to perturbations during dynamic movement (Manchester et al., 1989). If the high muscle coactivation is induced in order to maintain postural balance, this elevation should be observed even in static postural control and should be associated with the level of postural control ability.

Both static and dynamic postural controls are necessary during activities of daily living. Aging has been associated with deterioration in postural control, which manifests itself by an increase in postural sway (Era and Heikkinen, 1985), a decrease in reach distance (Duncan et al., 1992), and a decrease in capacity for locomotion (Oberg et al., 1993). Deterioration in these functions leads to a higher risk of falling, which may increase the number of bedridden persons (Lord et al., 1991; Duncan et al., 1992). However, the relationship between muscle coactivation and balance ability has not been clarified. Clarification of this relationship would be helpful to developing optimal rehabilitation strategies for older people.

The purpose of this study was to clarify the differences in muscle coactivation during postural control between older and young adults and to identify the characteristics of postural control strategies in older adults by investigating the relationship between muscle coactivation and postural control ability. We hypothesized that muscle coactivation during postural control in older adults is higher than that in young adults. We also hypothesized that muscle coactivation relates to postural control ability in young and older adults.

\section{Subjects and methods}

\subsection{Participants}

Forty-six healthy older adults (10 males, 36 females; age, $82.0 \pm 7.5$ years) and 34 healthy young adults (14 males, 20 females; age: $22.1 \pm 2.3$ years) participated in this study (Table1). Oral and written explanations of the study were offered to participants. Subjects were excluded if they had acute neurological impairment (stroke, Parkinson's disease, paresis of the lower limbs), severe cardiovascular disease, severe cognitive impairment: rapid dementia screening test score is of four points or less (Kalbe et al., 2003), persistent joint pain, or musculoskeletal impairment. 
Each subject gave informed consent indicating their agreement with the study protocol. This research was approved by the Ethical Review Board of Kyoto University Graduate School of Medicine, Kyoto, Japan.

\subsection{Testing procedures and protocol}

The postural tasks selected for testing consisted of static standing, functional reach, functional stability boundary (forward and backward), and gait because similar movements are performed frequently during activities of daily living.

\subsubsection{Postural sway}

Postural sway during static standing was measured with a force plate (Kistler 9286 force platform, Kistler Instruments Inc, Amherst, NY). Signals were sampled at $20 \mathrm{~Hz}$ and processed by a low pass filter (6 Hz cut off frequency). The participants were required to stand on a force plate with their feet together and then asked to gaze at a mark at eye level. Subjects were instructed to stand still as symmetrically as possible. Static standing balance was registered for a period of 10 seconds, from which the root mean square (RMS) area was calculated. EMG was measured for 3 seconds starting at the beginning of static standing.

\subsubsection{Functional stability boundary}

Functional stability boundary tasks were performed on the force plate (Slobounov et al., 1998). The subjects were instructed to stand with their heels positioned on a line $10 \mathrm{~cm}$ anterior to the posterior edge of the plate. The subjects were instructed to stand still for 5 seconds and then to shift their body weight first towards their toes and then towards their heels over the largest possible amplitude. They were further instructed to maintain full contact between their feet and the plate (avoiding toes off or heels off). For each direction (forward and backward), the subject maintained their posture for $3 \mathrm{sec}$ for EMG measurements, from which the averaged peak center of pressure (COP) displacement from the initial position was calculated. The COP displacement for each subject was normalized individually to the length of that subject's foot. 


\subsubsection{Functional reach}

The functional reach test (Duncan et al., 1990, 1992) measures the distance that subjects are able to reach forward while maintaining a fixed base. The position of the fingertip is determined with the shoulder of the subject flexed at $90^{\circ}$ along a wall. The subjects then were instructed to reach as far forward as possible without moving their feet, thus moving the center of gravity forward over a fixed base. Additionally, the subjects were instructed to keep their position for 3 seconds for EMG measurements. Functional reach was defined as the difference between arm's length and maximal forward reach.

\subsubsection{Gait}

Subjects were asked to perform walking trials at their preferred speed over a 12-m walkway. The examiner measured the time and the number of steps for the middle 10-m. A single trial was conducted following instruction. Walking speed $(\mathrm{m} / \mathrm{s})$ and step cadence (steps/min) were calculated as variables. EMG was analyzed for three gait cycles as determined from signals from the foot switch sensors (Noraxon USA Inc, Scottsdale, AZ) during the one gait trial.

\subsubsection{Additional physical function characteristics}

The timed up and go test (TUG) (Shumway-Cook et al., 2000) and the timed one-leg standing test for the dominant leg with eyes open were performed without EMG monitoring. The maximum duration of the one-leg standing test was set at 30 seconds.

\subsection{EMG recording}

EMG data were collected with the Telemyo 2400 (Noraxon USA Inc, Scottsdale, $A Z$ ). The skin of the dominant leg was shaved over the fibula head, tibialis anterior (TA), and soleus (SOL) (Melzer et al., 2001) and then washed with alcohol. Bipolar surface electrodes (Ambu, Blue sensor M, Denmark) with a $2.0 \mathrm{~cm}$ inter-electrode distance were placed on the skin around the probable motor point of the muscles (Hermens). The ground electrode was affixed to the skin over the fibula head of the dominant leg. The EMG data were sampled at $1500 \mathrm{~Hz}$.

EMG activity was recorded from the SOL and TA while the subjects were performing maximal voluntary contractions (MVC). The MVC of the SOL was 
obtained during maximal isometric plantar flexion, and maximal TA activation was recorded during maximal isometric dorsiflexion of the ankle at $90^{\circ}$ (anatomically neutral position). Strong verbal encouragement was given during every contraction to promote maximal effort. The EMG data from the MVCs were used to normalize the EMG amplitude (\%MVC) during the postural tasks.

\subsection{Muscle coactivation analysis}

The original raw EMG signal was band-pass filtered at 20-500 Hz. We computed the root mean-square amplitude of the signal using a 50-ms window. The EMG of each muscle was then expressed as a percentage of the EMG value during the MVC.

To evaluate the relative level of co-contraction of the tibialis anterior and soleus muscles, the co-contraction index $(\mathrm{Cl})$ was calculated using the method of Falconer and Winter (Falconer and Winter, 1985). Specifically, the following equation was used:

$$
\mathrm{CI}=\frac{2 I_{\text {ant }}}{I_{\text {Total }}} \times 100 \%
$$

Where $I_{a n t}$ is the area of the total antagonistic activity, calculated in accord with the following equation:

$$
I_{\mathrm{ant}}=\int_{t_{1}}^{t_{2}} \mathrm{EMG}_{\mathrm{TA}}(\mathrm{t}) \mathrm{d} t+\int_{t_{2}}^{t_{\mathrm{I}}} \mathrm{EMG}_{\mathrm{SOL}}(\mathrm{t}) \mathrm{d} t
$$

where $t 1$ to $t 2$ denotes the period during which the TA EMG is less than the SOL EMG and t2 to t3 donates the period during which the SOL EMG is less than the TA EMG), Itotal is the integral of the sum of TA and SOL EMG during performance of the task, calculated in accord with the following equation:

$$
I_{\text {total }}=\int_{t_{1}}^{t_{\mathrm{a}}}\left[\mathrm{EMG}_{\text {agon }}+\mathrm{EMG}_{\text {ant }}\right](t) \mathrm{d} t
$$

\subsection{Statistics}

SPSS (SPSS Inc, Tokyo, Japan) software was used for the data analysis. The test-retest intraday reliability of each EMG measurements without relocating the electrode was estimated by calculating intraclass correlation coefficients $\left(\mathrm{ICC}_{1.1}\right)$. 
Differences in physical function between young and older adults were tested using the Student's t-test for continuous variables and the Mann Whitney $U$ test for nonnormally distributed variables. Comparisons of the normalized EMG activity and cocontraction index between young and older adults were performed using the Mann Whitney $U$ test. The effect of gender differences in co-contraction index was examined with a $2 \times 2$ (gender $\times$ generation group) analysis of variance (ANOVA). The relationship between coactivation and physical function for each trial was analyzed by Spearman rank correlation. Statistical significance was set at $p<0.05$.

\section{Results}

The $\mathrm{ICCs}_{1.1}$ for $\mathrm{Cl}$ without relocating the electrode were as follows: $0.87(95 \% \mathrm{Cl}$ : 0.67 to 0.96$)$ for static standing, $0.95(95 \% \mathrm{Cl}: 0.84$ to 0.97$)$ for functional reach, 0.71 (95\% $\%$ Cl: 0.22 to 0.91$)$ for functional stability boundary (forward), 0.97 (95\% 0.92 to $0.99)$ for functional stability boundary (backward), and $0.86(95 \% \mathrm{Cl}: 0.57$ to 0.96$)$ for gait. These ICC values indicated 'good' to 'excellent' reliability for all measurements of Cl (Shrout and Fleiss, 1979).

The young adults had significantly better physical function than the older adults for all variables measured $(p<0.01$ ) (Table 2$)$. The elderly had significantly greater $\%$ MVC values in ankle muscles than the young adults for all measured tasks except for the SOL during functional reach $(p<0.01$, Table 3$)$.

The older adults had significantly higher $\mathrm{Cl}$ values than the young adults for standing, functional reach, functional stability boundary (forward), and gait ( $p<0.01$, Figure 1). The $\mathrm{Cl}$ for functional stability boundary (backward) tended to be higher in the older adults than in the young adults but the difference did not reach statistical significance $(p=0.072$ ). Two-way ANOVA identified no significant gender/generation interactions with respect to $\mathrm{Cl}$ for all tasks $(\mathrm{p}>0.05)$. Gender alone had no significant effect on $\mathrm{Cl}$ except for functional stability boundary (backward) $(\mathrm{p}<0.05)$.

In the older adults, Spearman rank correlation revealed a significant correlation between postural sway area and $\mathrm{Cl}$ during static standing $(\rho=0.42, p<0.05)$. Functional reach distance significantly correlated with $\mathrm{Cl}(\rho=-0.52, \mathrm{p}<0.05)$ (Figure 2). The functional stability boundary (forward) tended to correlate with $\mathrm{Cl}$ but did not reach statistical significance $(\rho=-0.33, p=0.06)$. In the young adults, no correlation between physical function and $\mathrm{Cl}$ was found. 


\section{Discussion}

Although previous studies have reported higher muscle coactivation during MVC, gait, and static standing in older adults compared with young adults (Klein et al., 2001; Melzer et al., 2001; Macaluso et al., 2002; Mian et al., 2006; Hortobagyi et al., 2009), no studies have quantified muscle coactivation during static and dynamic postural tasks in the elderly. The present study focused on quantifying muscle coactivation at the ankle joint under postural control and yielded two major findings: (1) Muscle coactivation at the ankle joint during postural control was higher in the elderly than in the young adults, and (2) the elderly who had less physical function during postural control tasks showed higher muscle coactivation at the ankle joints. These results, in turn, raise two alternative possibilities: either (1) older adults with deterioration in their postural control utilize muscle coactivation as a strategy to maintain their balance, or (2) high muscle coactivation in older adults leads to poor postural control.

Regarding static postural control, the data in the study by Meltzer et al. (2001) suggested higher coactivation in older adults than in young adults during static standing. Additionally, Manchaster et al. (1989) have demonstrated greater amounts of co-contraction of antagonists in older adults under perturbed conditions. These researchers described the greater co-contraction of antagonists with resultant ankle joint stiffness as a strategy to maintain postural stability.

In the present study, tibialis anterior activation during standing was greater in the older adults than in the young adults. During normal standing, the planter flexor muscles are recruited mainly as antigravity muscles at the ankle joint (Kendall and MacCreary, 1983) while the activity of the tibialis anterior usually remains low. The greater tibialis anterior activity in the older adults probably caused the observed increase in muscle coactivation in this study.

The results of the dynamic postural control tasks, i.e., functional reach and functional stability boundary, suggest that muscle coactivation becomes stronger in older adults who cannot sufficiently move their center of pressure within their base of support. Hortobagyi and DeVita (2000), have reported increased coactivation of antagonistic muscles during stair descending in older adults compared with young adults, which they interpreted as an indication that the older adults had increased muscle coactivation in order to stiffen the joints of their lower limbs. Functional reach and the functional stability boundary during forward motion involve a forward 
movement of the center of pressure, which requires increased plantar flexor torque at the ankle joint to control posture. Therefore, the tibialis anterior plays the role of antagonist in these tasks. In the present study, during functional reach and functional stability boundary for forward motion, the EMG activity of the tibialis anterior reached almost $20 \%$ of MVC in the older adults, probably to increase the stiffness of their ankle joint. The elevated tibialis anterior activity may have caused the increase in muscle coactivation in a similar manner as during static postural control.

Our gait measurements showed higher muscle coactivation during walking in the older adults than in the young adults. This difference indicates that aging induces not only a decline in walking ability (e.g., decrease in gait velocity, increase in step variability) but also a change in central nervous system control of dynamic movement. Recent studies have also reported higher muscle coactivation at the ankle joint during walking in older adults than in young adults (Schmitz et al., 2008; Hortobagyi et al., 2009). In these studies, high muscle coactivation during walking was interpreted as being a means of enhancing ankle joint stability. Chambers and Cham (2007) have investigated the relationship between muscle co-contraction and slips during gait measurements. The subjects who walked with higher ankle muscle cocontraction were predisposed to experience less severe slips when encountering an unexpected slippery floor (Chambers and Cham, 2007). Additionally, anticipation of a slippery surface resulted in more muscle co-contraction at the ankle and knee. These observations support the hypothesis that the elderly have enhanced muscle coactivation to compensate for an age-associated decline in postural control ability.

Our study also found no significant correlation between gait velocity and muscle cocactivation during walking. Muscle coactivation during walking seems to be independent from gait velocity. Hortobagyi et al. (2009) have investigated muscle coactivation during walking at different velocities and reported that muscle coactivation was not affected by gait velocity in older people. This finding suggests that older adults probably can utilize a high muscle coactivation strategy during walking regardless of gait velocity.

In previous studies, high muscle co-contraction has been described as a strategy to stiffen the joint and enhance stability (Hortobagyi and DeVita, 2000; Hortobagyi et al., 2009). The purpose of increasing joint stiffness is to compensate for the many neuromotor impairments associated with aging, including reduced muscle strength (Hortobagyi et al., 1995), slower rate of tension development 
(Thelen et al., 1996), reduced proportion of fast muscle fibers (Larsson et al., 1979), miscued limb positioning (Skinner et al., 1984), and fear of falling (Okada et al., 2001). Calorane and Cafarelli (1992) have measured muscle activation in the biceps femoris during knee extension and found that as knee extensor strength increased, biceps femoris activity also increased. This finding indicates that greater effort to extend the knee joint induces increased muscle coactivation in the antagonist muscle. In the present study, the elderly showed higher muscle activity than the young adults in most of the measured tasks. Because of age-related decreases in their muscular strength, older adults need to recruit more muscle fibers to accomplish a specific postural task. This increased muscle effort possibly led to the higher muscle coactivation that we observed.

Increased muscle coactivation may be a necessary change to compensate for the poor postural control accompanying healthy aging. However, strong coactivation of agonist and antagonist muscles should reduce the performance of agonist muscles (Carolan and Cafarelli, 1992; Pereira and Goncalves, 2010) as well as increasing the energetic cost of transport (Mian et al., 2006), thereby inducing fatigue and potentially increasing the risk of falling (Hortobagyi et al., 2009). If older adults employ the coactivation strategy for postural control, probable consequences would include poor performance and increased risk of falling, especially in situations difficult to deal with by this strategy. In clinical settings, efforts to determine the proper level of coactivation, corresponding to personal and environmental situations, should be targeted especially towards those older patients who have suffered deterioration in their postural control ability.

Limitations of our study include that our study data did not clarify the relationship between muscle coactivation and joint kinematics. In addition, our study provided no information on a postural strategy for the knee or hip joint because it focused on the ankle joint. Finally, the lack of longitudinal data precludes an examination of the causal relationship between deterioration in postural control and muscle coactivation. Further studies are needed to examine muscle coactivation strategy in postural tasks for the elderly in greater detail.

\section{Conclusions}

Our study clarified that older adults show greater muscle coactivation during postural control compared with young adults. Moreover, muscle coactivation was 
significantly higher in older adults with low postural control ability than in older adults with high postural control ability. Increased muscle coactivation could be a necessary change to compensate for the deterioration in postural control accompanying healthy aging. However, further research is needed to clarify in greater detail the positive and negative effects of muscle coactivation on postural control.

Conflict of interest statement: None.

\section{Acknowledgements}

We would like to thank all volunteers for participating in this study.

\section{References}

Baratta, R., Solomonow, M., Zhou, B.H., Letson, D., Chuinard, R., D'Ambrosia, R., 1988. Muscular coactivation. The role of the antagonist musculature in maintaining knee stability. Am. J. Sports Med. 16, 113-122.

Carolan, B., Cafarelli, E., 1992. Adaptations in coactivation after isometric resistance training. J. Appl. Physiol. 73, 911-917.

Chambers, A.J., Cham, R., 2007. Slip-related muscle activation patterns in the stance leg during walking. Gait Posture 25, 565-572.

Dierick, F., Domicent, C., Detrembleur, C., 2002. Relationship between antagonistic leg muscles co-contractions and body centre of gravity mechanics in different level gait disorders. J. Electromyogr. Kinesiol. 12, 59-66.

Duncan, P., Weiner, D., Chandler, J., Studenski, S., 1990. Functional reach: a new clinical measure of balance. J. Gerontol. 45, M192-M197.

Duncan, P., Studenski, S., Chandler, J., Prescott, B., 1992. Functional reach: predictive validity in a sample of elderly male veterans. J. Gerontol. 47, M93M98.

Era, P., Heikkinen, E., 1985. Postural sway during standing and unexpected disturbance of balance in random samples of men of different ages. J. Gerontol. 40, 287-295.

Falconer, K., Winter, D.A., 1985. Quantitative assessment of co-contraction at the ankle joint in walking. Electromyogr. Clin. Neurophysiol. 25, 135-149. 
Fujii, S., Kudo, K., Ohtsuki, T., Oda, S., 2009. Tapping performance and underlying wrist muscle activity of non-drummers, drummers, and the world's fastest drummer. Neurosci. Lett. 459, 69-73.

Hermens, H., SENIAM. http://www.seniam.org/

Hortobagyi, T., DeVita, P., 2000. Muscle pre- and coactivity during downward stepping are associated with leg stiffness in aging. J. Electromyogr. Kinesiol. 10, 117-126.

Hortobagyi, T., Zheng, D., Weidner, M., Lambert, N.J., Westbrook, S., Houmard, J.A., 1995. The influence of aging on muscle strength and muscle fiber characteristics with special reference to eccentric strength. J. Gerontol. A: Biol. Sci. Med. Sci. 50, B399-B406.

Hortobagyi, T., Solnik, S., Gruber, A., Rider, P., Steinweg, K., Helseth, J., DeVita, P., 2009. Interaction between age and gait velocity in the amplitude and timing of antagonist muscle coactivation. Gait Posture 29, 558-564.

Kalbe, E., Calabrese, P., Schwalen, S., Kessler, J., 2003. The Rapid Dementia Screening Test (RDST): a new economical tool for detecting possible patients with dementia. Dement. Geriatr. Cogn. Disord. 16, 193-199.

Kendall, H., MacCreary, E., 1983. Testing and Functions, ed. 3. Williams \& Wilkins, Baltimore.

Klein, C.S., Rice, C.L., Marsh, G.D., 2001. Normalized force, activation, and coactivation in the arm muscles of young and old men. J. Appl. Physiol. 91, 1341-1349.

Larsson, L., Grimby, G., Karlsson, J., 1979. Muscle strength and speed of movement in relation to age and muscle morphology. J. Appl. Physiol. 46, 451-456.

Lord, S.R., Clark, R.D., Webster, I.W., 1991. Physiological factors associated with falls in an elderly population. J. Am. Geriatr. Soc. 39, 1194-1200.

Macaluso, A., Nimmo, M., Foster, J., Cockburn, M., McMillan, N., De Vito, G., 2002. Contractile muscle volume and agonist-antagonist coactivation account for differences in torque between young and older women. Muscle Nerve 25, 858863.

Manchester, D., Woollacott, M., Zederbauer-Hylton, N., Marin, O., 1989. Visual, vestibular and somatosensory contributions to balance control in the older adult. J. Gerontol. 44, M118-M127. 
Melzer, I., Benjuya, N., Kaplanski, J., 2001. Age-related changes of postural control: effect of cognitive tasks. Gerontology 47, 189-194.

Mian, O.S., Thom, J.M., Ardigo, L.P., Narici, M.V., Minetti, A.E., 2006. Metabolic cost, mechanical work, and efficiency during walking in young and older men. Acta Physiol. (Oxf.) 186, 127-139.

Oberg, T., Karsznia, A., Oberg, K., 1993. Basic gait parameters: reference data for normal subjects, 10-79 years of age. J. Rehabil. Res. Dev. 30, 210-223.

Okada, S., Hirakawa, K., Takada, Y., Kinoshita, H., 2001. Relationship between fear of falling and balancing ability during abrupt deceleration in aged women having similar habitual physical activities. Eur. J. Appl. Physiol. 85, 501-506.

Pereira, M.P., Goncalves, M., 2010. Muscular coactivation (CA) around the knee reduces power production in elderly women. Arch. Gerontol. Geriatr. ahead of print: doi:10.1016/j.archger.2010.04.024

Schmitz, A., Silder, A., Heiderscheit, B., Mahoney, J., Thelen, D.G., 2008. Differences in lower-extremity muscular activation during walking between healthy older and young adults. J. Electromyogr. Kinesiol. 19, 1085-1091.

Shrout, P.E., Fleiss, J.L., 1979. Intraclass correlations: uses in assessing rater reliability. Psychol. Bull. 86, 420-428.

Shumway-Cook, A., Brauer, S., Woollacott, M., 2000. Predicting the probability for falls in community-dwelling older adults using the Timed Up \& Go Test. Phys. Ther. 80, 896-903.

Skinner, H.B., Barrack, R.L., Cook, S.D., 1984. Age-related decline in proprioception. Clin. Orthop. Relat. Res. (184), 208-211.

Slobounov, S.M., Moss, S.A., Slobounova, E.S., Newell, K.M., 1998. Aging and time to instability in posture. J. Gerontol. A: Biol. Sci. Med. Sci. 53, B71-B78.

Solomonow, M., Baratta, R., Zhou, B.H., D'Ambrosia, R., 1988. Electromyogram coactivation patterns of the elbow antagonist muscles during slow isokinetic movement. Exp. Neurol. 100, 470-477.

Thelen, D.G., Schultz, A.B., Alexander, N.B., Ashton-Miller, J.A., 1996. Effects of age on rapid ankle torque development. J. Gerontol. A: Biol. Sci. Med. Sci. 51, M226-M232. 
Table 1. Physical characteristics of the subjects, $n$, mean \pm S.D., or $\%$

\begin{tabular}{lll}
\hline Parameters & Elderly & Young \\
\hline Number & 46 & 34 \\
Age (years) & $82.0 \pm 7.5$ & $22.1 \pm 2.3$ \\
Female & 58.8 & 78.3 \\
Height $(\mathrm{cm})$ & $151.3 \pm 6.8$ & $164.6 \pm 7.9$ \\
Weight $(\mathrm{kg})$ & $51.9 \pm 6.6$ & $56.7 \pm 8.0$ \\
\hline
\end{tabular}


Table 2. Postural control ability of subjects, $n$, mean \pm S.D.

\begin{tabular}{lccll}
\hline Physical function & Elderly & Young & $95 \% \mathrm{Cl}$ & $\mathrm{p}<$ \\
\hline Number & 46 & 34 & & \\
Postural sway area $\left(\mathrm{cm}^{2}\right)$ & $1.7 \pm 1.0$ & $0.9 \pm 0.4$ & $0.48-1.19$ & $0.001^{*}$ \\
$\begin{array}{l}\text { Functional reach (cm) } \\
\text { Functional stability boundary (forward) }\end{array}$ & $20.9 \pm 7.9$ & $37.0 \pm 5.4$ & $-19.1--12.9$ & $0.001^{*}$ \\
& $25.5 \pm 9.7$ & $36.9 \pm 5.5$ & & $0.001 \dagger$ \\
Functional stability boundary (backward) & $(\%)$ & & \\
& $18.4 \pm 4.6$ & $23.9 \pm 5.4$ & & $0.001 \dagger$ \\
Gait speed (m/s) & $0.9 \pm 0.4$ & $1.2 \pm 0.2$ & $-0.47--0.12$ & $0.001^{*}$ \\
TUG test (sec) & $11.4 \pm 6.4$ & $6.0 \pm 0.7$ & $3.16-7.60$ & $0.001^{*}$ \\
One-leg standing (sec) & $9.3 \pm 11.2$ & $30 \pm 0.0$ & & $0.001 \dagger$ \\
\hline
\end{tabular}

Notes: *Student's t-test; †Mann Whitney U-test 
Table 3. Mean normalized EMG activity (\%MVC) during measured tasks, $n$, mean \pm S.D.

\begin{tabular}{lllll}
\hline Tasks & Muscle & Elderly & Young & $\mathrm{p}<$ \\
\hline Standing (\%) & TA & $19.4 \pm 19.3$ & $2.1 \pm 2.6$ & 0.01 \\
& SOL & $28.6 \pm 20.4$ & $12.5 \pm 8.2$ & 0.01 \\
Functional reach (\%) & TA & $18.6 \pm 19.7$ & $4.8 \pm 4.2$ & 0.01 \\
& SOL & $45.3 \pm 23.3$ & $43.1 \pm 26.7$ & 0.36 \\
Functional stability boundary (forward) & $(\%)$ & & \\
& TA & $19.4 \pm 17.0$ & $6.0 \pm 8.0$ & 0.01 \\
& SOL & $52.0 \pm 22.9$ & $37.6 \pm 21.5$ & 0.01 \\
Functional stability boundary (backward) & $(\%)$ & & \\
& TA & $49.6 \pm 22.1$ & $28.1 \pm 12.2$ & 0.01 \\
& SOL & $37.8 \pm 17.8$ & $17.6 \pm 11.5$ & 0.01 \\
Gait (\%) & TA & $35.4 \pm 19.0$ & $12.4 \pm 4.6$ & 0.01 \\
& SOL & $48.1 \pm 22.1$ & $28.8 \pm 14.0$ & 0.01
\end{tabular}

Notes: Mann Whitney U-test was used for comparison of the \%MVC; 

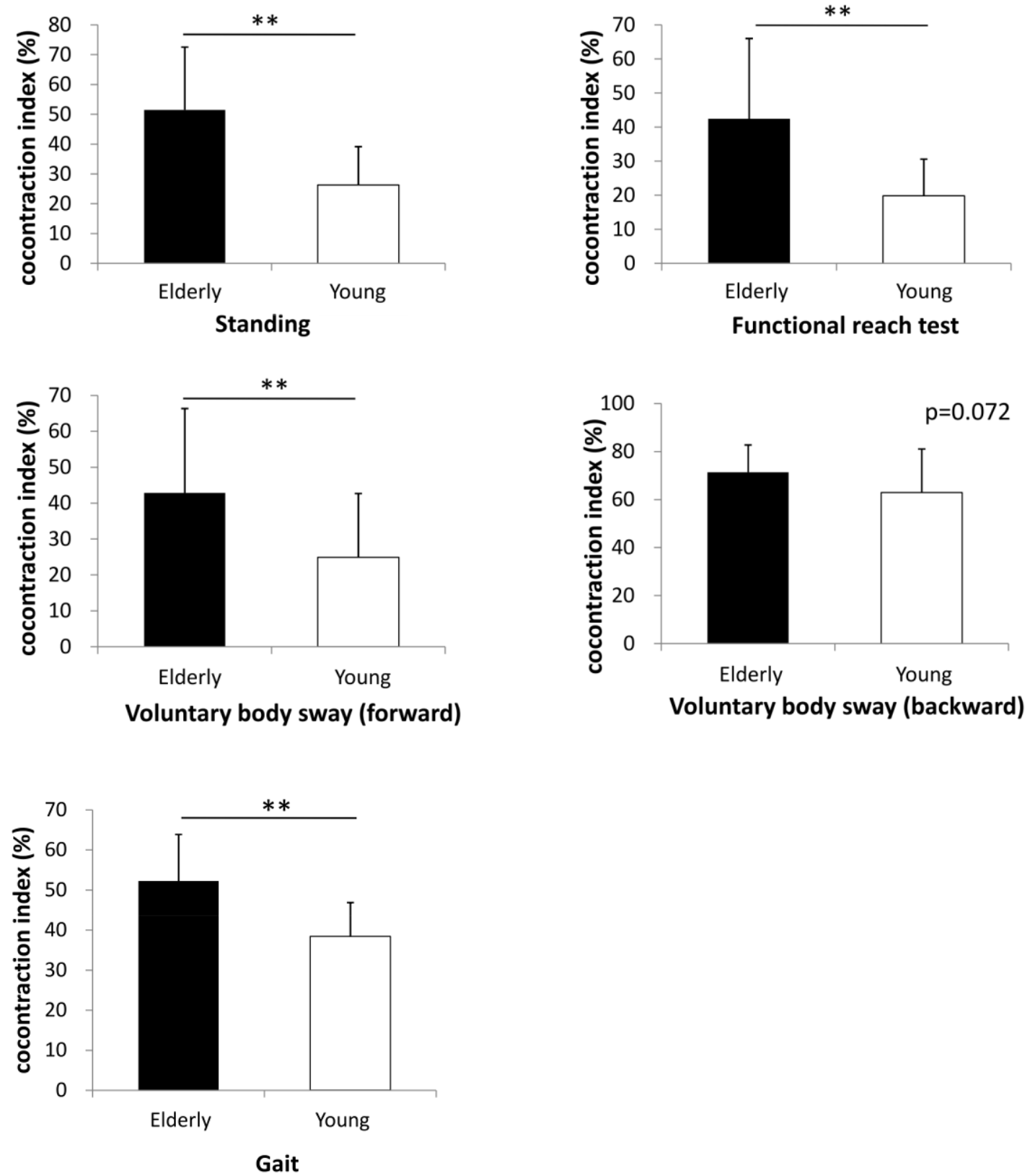

Figure 1.

Comparisons of the co-contraction index $(\mathrm{Cl})$ between older (filled bars) and young adults (open bars) during the measured tasks. The Mann Whitney $U$ test was used for the $\mathrm{Cl}$ comparisons. $\left({ }^{*} \mathrm{p}<0.05,{ }^{* *} \mathrm{p}<0.01\right)$ 

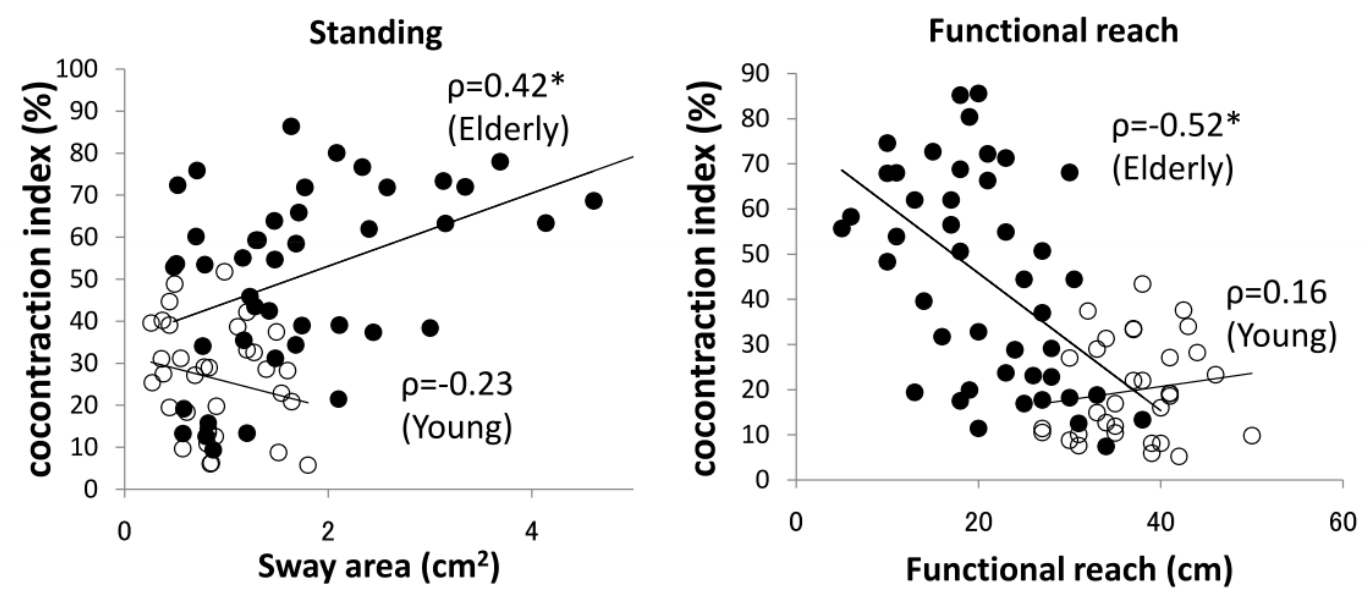

Functional stability boundary (forward)

Functional stability boundary (backward)
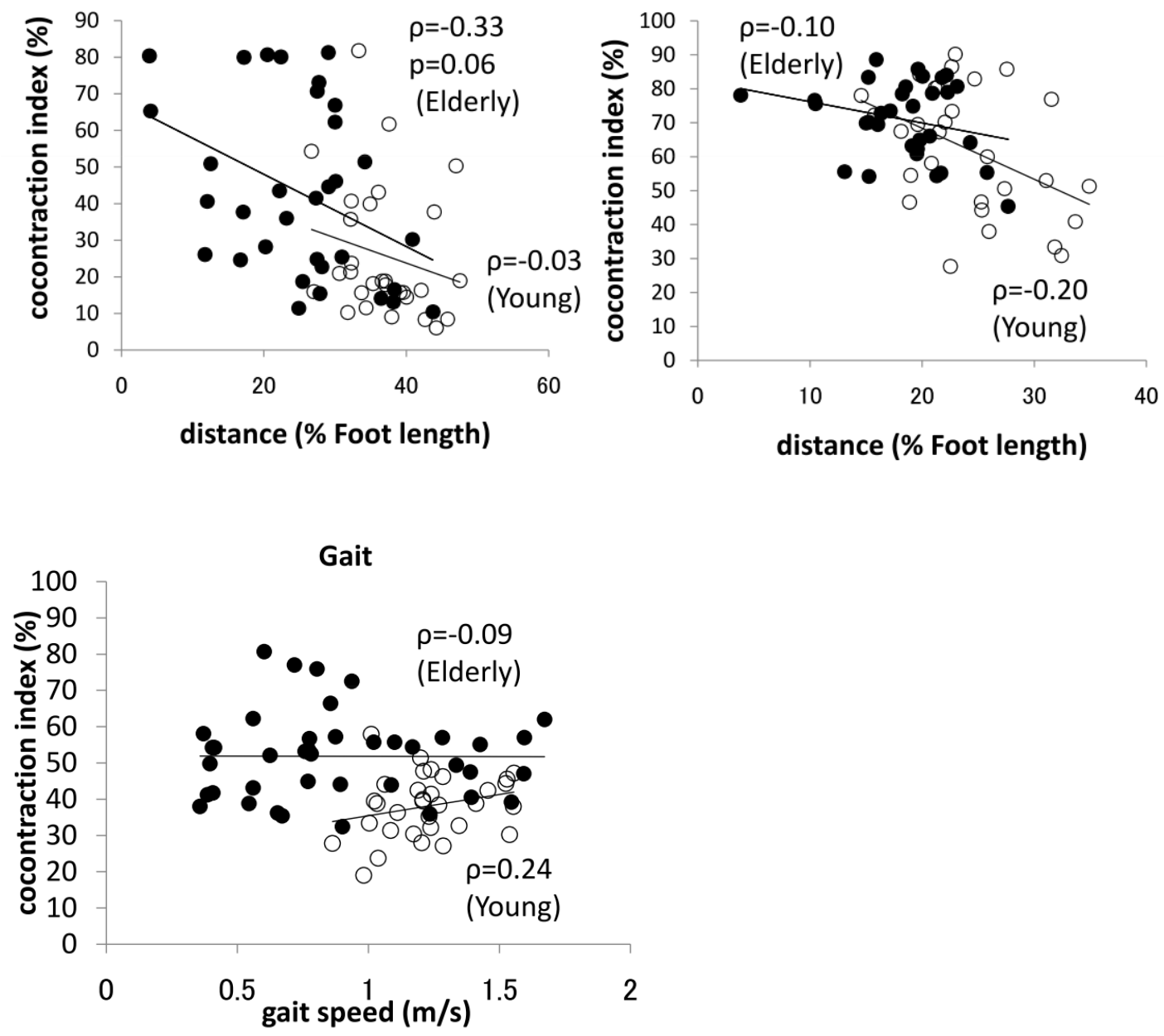

Figure 2. Correlations between postural control ability and co-contraction index $(\mathrm{Cl})$. Filled circles show older adults and open circles show young adults. Spearman rank correlation was used for statistical analysis. ( $\rho$ : correlation coefficient, ${ }^{*} p<0.05$ ) 